\title{
J. BOUZITAT
}

\section{Brèves communications : "Note sur la méthode de Wolfe en programmation qradratique"}

Revue française d'automatique, d'informatique et de recherche opérationnelle. Recherche opérationnelle, tome 11, n 2 (1977), p. 239-241.

<http://www.numdam.org/item?id=RO_1977_11_2_239_0>

(C) AFCET, 1977, tous droits réservés.

L'accès aux archives de la revue « Revue française d'automatique, d'informatique et de recherche opérationnelle. Recherche opérationnelle » implique l'accord avec les conditions générales d'utilisation (http://www.numdam.org/ legal.php). Toute utilisation commerciale ou impression systématique est constitutive d'une infraction pénale. Toute copie ou impression de ce fichier doit contenir la présente mention de copyright.

\section{Numdam}

Article numérisé dans le cadre du programme

Numérisation de documents anciens mathématiques

http://www.numdam.org/ 


\title{
NOTE SUR LA MÉTHOdE DE WOLFE EN PROGRAMMATION QUADRATIQUE
}

\author{
par J. BouZitaT
}

Dans son article «The Simplex Method for quadratic Programming » (Econometrica, Vol. 27, n³ , pp. 382-398 - juillet 1959), Philip Wolfe présente une méthode de résolution des programmes quadratiques à contraintes linéaires où la fonction à minimiser est convexe.

Cette méthode utilise les conditions de Kuhn et Tucker, qui sont alors nécessaires et suffisantes et qui, à l'exception des relations de complémentarité (ou d'exclusion), sont linéaires par rapport à l'ensemble des variables primales et duales. Cela conduit à en chercher des « solutions de base » par une méthode généralisant la méthode du Simplexe, avec une restriction qui impose le respect des relations de complémentarité.

Plus précisément, Wolfe introduit des variables artificielles positives ou nulles dont il se propose de minimiser la somme en appliquant l'algorithme du Simplexe, mais en imposant constamment la nullité d'une variable dans chaque couple de variables complémentaires. L'optimum est atteint quand toutes les variables artificielles sont annulées.

Cependant, en raison de la restriction imposée par le respect des relations de complémentarité, il n'est pas sûr a priori que la méthode permette toujours d'atteindre l'optimum quand il existe. Certains exemples ont en effet conduit à penser que le processus pouvait se bloquer pour une valeur strictement positive de la somme des variables artificielles, même quand cette somme a un minimum nul.

En complétant l'étude théorique de l'efficacité de la méthode, telle qu'elle est faite dans l'article cité, nous avons montré qu'un tel blocage n'est qu'apparent, car il s'accompagne nécessairement d'une dégénérescence qui permet de poursuivre le processus par un pivotage dans lequel la somme des variables artificielles reste constante, mais la fonction à minimiser du problème initial décroît strictement.

La convergence du processus vers l'optimum est ainsi assurée en un nombre fini de pas. Cela dispense de recourir à la " forme longue » que Wolfe avait proposée pour résoudre les cas de blocage de la «forme courte». 
Cependant la forme longue de la méthode de Wolfe peut conserver un certain intérêt pour traiter des problèmes paramétriques. Sur ce point aussi, nous avons pu' compléter l'étude théorique de l'efficacité de la méthode grâce à des remarques analogues à celles qui ont été faites à propos de la forme courte.

Ces différentś résultats seront établis et développés dans un prochain article.

Exemple (proposé par Richard Cottle).

Minimiser $f(x)=-10 x_{1}-4 x_{2}+\left(x_{1}-2 x_{2}\right)^{2}$ sous les contraintes

$$
\left\{\begin{array}{r}
x_{1}+x_{2} \leqslant 6 \\
4 x_{1}+x_{2} \leqslant 18 \\
x_{1}, \quad x_{2} \geqslant 0
\end{array}\right.
$$

Conditions de Kuhn et Tucker (nécessaires et suffisantes).

$$
\left\{\begin{array}{rrrr}
-10+2 x_{1}-4 x_{2}+y_{3}+4 y_{4}-y_{1} & =0 \\
-4-4 x_{1}+8 x_{2}+y_{3}+y_{4}-y_{2} & =0 \\
x_{1}+x_{2} & & +x_{3}= & =6 \\
4 x_{1}+x_{2} & & x_{4}= & 18 \\
x_{i} \geqslant 0, y_{i} \geqslant 0, x_{i} y_{i}=0, & \forall i \in\{1,2,3,4\}
\end{array}\right.
$$

Problème de Wolfe (forme courte).

Minimiser $\varphi(z)=z_{1}+z_{2}$ sous les contraintes

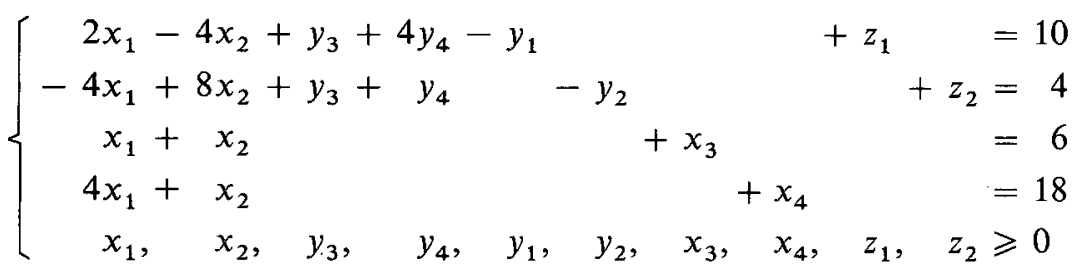

et sous les conditions de complémentarité $x_{i} y_{i}=0, \forall i \in\{1,2,3,4\}$.

Compte tenu des contraintes, la fonction économique peut être mise sous la forme suivante :

$$
2 f(x)=-10 x_{1}-4 x_{2}-6 y_{3}-18 y_{4}+\sum_{i=1}^{4} x_{i} y_{i}-x_{1} z_{1}-x_{2} z_{2} .
$$

Cette expression se prête bien au calcul de $f(x)$ à chaque pas de l'algorithme. 
Tableaux de calcul (réduits).

\begin{tabular}{c|rrrrrr|r} 
& $x_{1}$ & $x_{2}$ & $y_{3}$ & $y_{4}$ & $y_{1}$ & $y_{2}$ & \\
\hline$\varphi(z)$ & -2 & 4 & 2 & 5 & -1 & -1 & 14 \\
\hline$z_{1}$ & 2 & -4 & 1 & 4 & -1 & 0 & 10 \\
$z_{2}$ & -4 & 8 & 1 & 1 & 0 & -1 & 4 \\
$x_{3}$ & 1 & 1 & 0 & 0 & 0 & 0 & 6 \\
$x_{4}$ & 4 & 1 & 0 & 0 & 0 & 0 & 18 \\
\hline $2 f(x)$ & 10 & 4 & 6 & 18 & 0 & 0 & 0
\end{tabular}

\begin{tabular}{c|cccccc|c} 
& $x_{1}$ & $z_{2}$ & $y_{3}$ & $y_{4}$ & $y_{1}$ & $y_{2}$ & \\
\hline$\varphi(z)$ & 0 & $-1 / 2$ & $3 / 2$ & $9 / 2$ & -1 & $-1 / 2$ & 12 \\
\hline$z_{1}$ & 0 & $1 / 2$ & $3 / 2$ & $9 / 2$ & -1 & $-1 / 2$ & 12 \\
$x_{2}$ & $-1 / 2$ & $1 / 8$ & $1 / 8$ & $1 / 8$ & 0 & $-1 / 8$ & $1 / 2$ \\
$x_{3}$ & $3 / 2$ & $-1 / 8$ & $-1 / 8$ & $-1 / 8$ & 0 & $1 / 8$ & $11 / 2$ \\
$x_{4}$ & $9 / 2-1 / 8$ & $-1 / 8$ & $-1 / 8$ & 0 & $1 / 8$ & $35 / 2$ \\
\hline $2 f(x)$ & 12 & $-1 / 2$ & $11 / 2$ & $35 / 2$ & 0 & $1 / 2$ & -2
\end{tabular}

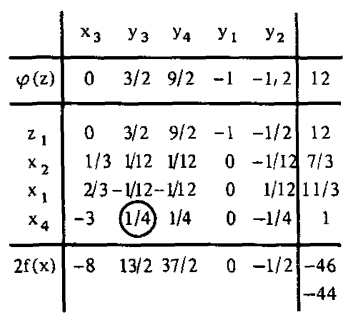

\begin{tabular}{c|ccccc|c} 
& $x_{3}$ & $x_{4}$ & $y_{4}$ & $y_{1}$ & $y_{2}$ & \\
\hline$\varphi(z)$ & 18 & -6 & 3 & -1 & 1 & 6 \\
\hline$z_{1}$ & 18 & -6 & 3 & -1 & 1 & 6 \\
$x_{2}$ & $4 / 3$ & $-1 / 3$ & 0 & 0 & 0 & 2 \\
$x_{1}$ & $-1 / 3$ & $1 / 3$ & 0 & 0 & 0 & 4 \\
$y_{3}$ & -12 & 4 & 1 & 0 & -1 & 4 \\
\hline $2 f(x)$ & 70 & -26 & 12 & 0 & 6 & -72 \\
& & & & & -24
\end{tabular}

\begin{tabular}{c|ccccc|c} 
& $x_{3}$ & $x_{4}$ & $z_{1}$ & $y_{1}$ & $y_{2}$ & \\
\hline$\varphi(z)$ & 0 & 0 & -1 & 0 & 0 & 0 \\
\hline$y_{4}$ & 6 & -2 & $1 / 3$ & $-1 / 3$ & $1 / 3$ & 2 \\
$x_{2}$ & $4 / 3$ & $-1 / 3$ & 0 & 0 & 0 & 2 \\
$x_{1}$ & $-1 / 3$ & $1 / 3$ & 0 & 0 & 0 & 4 \\
$y_{3}$ & -18 & 6 & $-1 / 3$ & $1 / 3$ & $-4 / 3$ & 2 \\
\hline $2 f(x)$ & -2 & -2 & -4 & 4 & 2 & -96 \\
& & & & &
\end{tabular}

La solution unique du problème est ainsi atteinte :

$$
\left\{\begin{array}{l}
\bar{x}_{1}=4 \\
\bar{x}_{2}=2
\end{array} \quad f(\bar{x})=-48\right.
$$

Remarques sur les tableaux de calcul.

L'élément encerclé dans chaque tableau autre que le dernier est le pivot.

Dès qu'une variable artificielle sort de la base, elle peut être supprimée.

La partie linéaire de la fonction économique, sous sa seconde forme, est calculée par l'algorithme du Simplexe. La partie quadratique (réduite aux termes $-x_{1} z_{1}-x_{2} z_{2}$ pour $2 f(x)$, en vertu des conditions de complémentarité) se calcule très simplement et, quand elle n'est pas nulle, sa valeur est inscrite sous la valeur de la partie linéaire.

Le second tableau de calcul conduit à un blocage apparent parce que $y_{3}$ et $y_{4}$ ne doivent pas être introduites dans la base, où $x_{3}$ et $x_{4}$ figurent avec des valeurs non nulles et devraient demeurer. Mais ce blocage n'est qu'apparent parce que $x_{1}$ peut être introduite dans la base et que cette introduction, sans modifier la valeur de $\varphi(z)$, conduit à une décroissance stricte de $f(x)$.

Dans l'exemple considéré, le processus se poursuit et s'achève sans autre blocage apparent. 